\title{
Novel biodegradable HSAM nanoparticle for drug delivery
}

\author{
BIN LIU, SHIQUAN JIANG, WANDI ZHANG, FEI YE, YU-HANG WANG, \\ JOSEPHINE WU and DAVID Y.ZHANG
}

Department of Pathology, Mount Sinai School of Medicine, New York, NY 10029, USA

Received October 20, 2005; Accepted December 22, 2005

\begin{abstract}
The systemic pharmacological treatment of disease is limited by severe toxicity to normal organs/tissue. Therefore, various delivery vehicles have been designed to carry therapeutic drugs to their target tissues. We designed a novel vehicle formed by the interaction of biotins in a DNA (polymer) with avidins (crosslink), resulting in a porous particle. This self-assembled (HSAM) nanoparticle vehicle has been tested in our laboratory both in vitro and in vivo for its ability to carry doxorubicin, a widely used anticancer drug with a high toxicity to normal organs. Doxorubicin binds to the nanoparticle by intercalating into the DNA strands that are later degraded by nucleases released from cancer cells. Our results showed that $1.1 \mu \mathrm{g}$ of HSAM DNA can carry $1 \mu \mathrm{g}$ of doxorubicin, and the doxorubicin-bound HSAM nanoparticle can still be degraded by nucleases (BAL-31 and DNase I). The HSAM nanoparticle carrying doxorubicin can efficiently inhibit cancer cell growth in vitro and in a murine model. Furthermore, this nanoparticle is able to deliver up to $180 \mathrm{ng} / \mathrm{mg}$ of doxorubicin to the target tumor tissue, which is 15 -fold above the systemic toxicity dose $(12 \mathrm{mg} / \mathrm{kg})$. These results suggest that the HSAM nanoparticle is both biocompatible and biodegradable, making it a valuable vehicle for drug delivery in cancer treatment.
\end{abstract}

\section{Introduction}

The continuous development of new drug delivery systems is driven by the need to maximize therapeutic activity while minimizing negative side effects. Anticancer drugs, such as doxorubicin and carboplatin, are widely used in cancer chemotherapy. Besides their low water solubility, the major drawbacks of these drugs are the acute toxicity to normal tissue and inherent multi-drug resistance effect. To reduce the acute toxicity of the free drugs and improve their therapeutic efficacy, various delivery vehicles or systems

Correspondence to: Dr David Y. Zhang, Molecular Pathology Laboratory, Mount Sinai School of Medicine, Box 1122, One Gustave L. Levy Place, New York, NY 10029, USA

E-mail: david.zhang@mssm.edu

Key words: polymer, drug delivery, DNA, avidin have been designed, including the liposome (1-3), polymeric micelle systems $(2,4)$ and dendrimers $(5)$.

Although DNA has been used as a therapeutic agent in gene replacement (gene therapy), antisense, siRNA and ribozyme, it has not been used as a drug delivery system to carry toxic drugs to the target organs. A DNA complex can serve as an excellent drug delivery system with several significant advantages: 1) it is a natural component of the human body and, therefore, less harmful to the body and organs (i.e. excellent biocompatibility); 2) it can be efficiently degraded in the body by various nucleases and, therefore, minimize retention problems, i.e. excellent biodegradability; 3) because of its biodegradability, release of the bound drug can be achieved in the target organ; 4) many drugs, such as doxorubicin, naturally bind to DNA as part of their biological mechanisms and would, therefore, bind to the DNA complex without further modification; 5) the binding capacity of the DNA complex can be significantly large; and 6) it is easy to design, manipulate and manufacture. The goal of this study is to engineer a DNA complex using biotinylated DNA and avidin to form a porous DNA structure that can be used as a vehicle to carry an anticancer agent to cancer tissue. In this report, we used doxorubicin, a widely used anticancer drug, as a model system to demonstrate its ability to carry doxorubicin and inhibit squamous cancer cell growth, which is a deadly disease that claims thousands of lives each year.

\section{Materials and methods}

Formation of the HSAM nanoparticle. HSAM nanoparticles were formed in $70-\mu 1$ reactions in the presence of $3 \times 10^{14}$ molecules $(30 \mu \mathrm{g})$ of avidin (Sigma-Aldrich, Berlin, Germany) and $4 \times 10^{14}$ molecules $(6.6 \mu \mathrm{g})$ of biotinylated HSAM DNA with an avidin to HSAM DNA molar ratio of 3:4. The HSAM DNA was a 31-nucleotide long sequence (5'-Taaccaaag agcaacTacacgaattctcgaT-3') that contained three biotins at positions 1, 16, and 31 (capitalized) (Gene Link, Hawthorne, NY). The mixture of avidin and HSAM DNA was incubated at room temperature for $1 \mathrm{~h}$ with shaking at $120 \mathrm{rpm}$.

Binding doxorubicin to HSAM particles. A doxorubicin solution $(30 \mu \mathrm{l})$, prepared by the addition of $10 \mathrm{mg}$ of doxorubicin (Sigma) to $2 \mathrm{ml}$ of dimethylsulfoxide and $2 \mathrm{ml}$ of absolute ethanol, was added to $600 \mu 1$ of HSAM nanoparticle (containing $55 \mu \mathrm{g}$ of HSAM DNA) as described above. The doxorubicin-HSAM nanoparticle mixture was incubated for $1 \mathrm{~h}$, centrifuged at 3,800 rpm for $3 \mathrm{~min}$, and the 


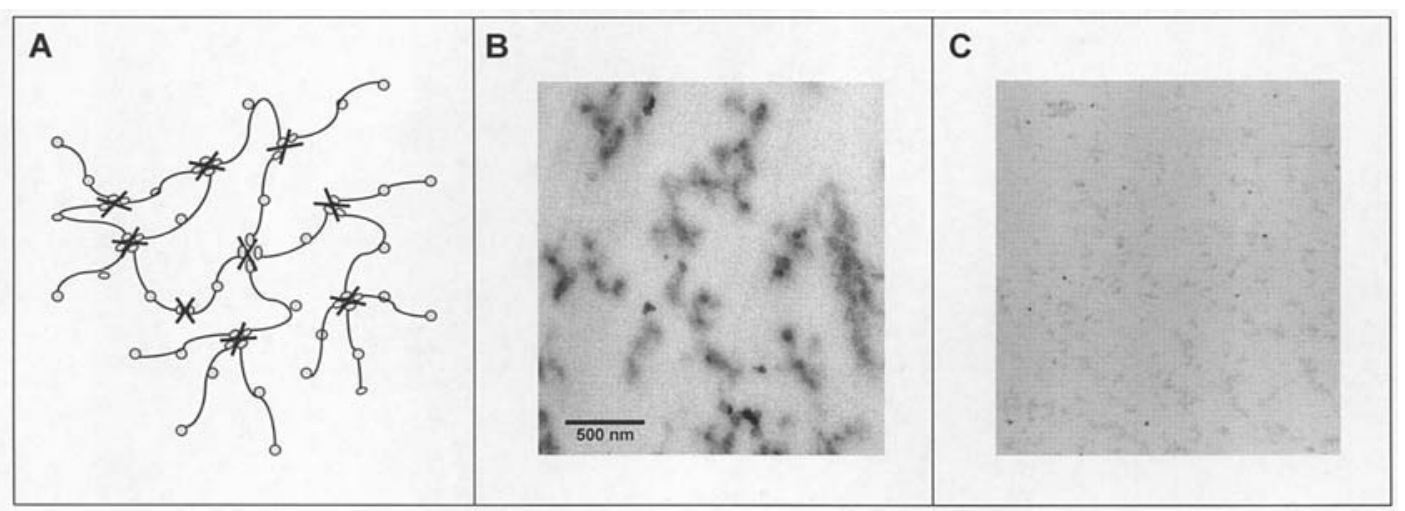

Figure 1. HSAM nanoparticles. (A) Schematic representation of the self-assembled HSAM nanoparticle. Each HSAM nanoparticle is composed of HSAM DNA (curved line) incorporated with three biotins (small circle) and avidins (cross). The HSAM DNA serves as a polymer, and avidin bridges the HSAM DNA together. (B) A representative electronic microscopic image of HSAM nanoparticles. HSAM nanoparticles of various shape and size were identified, ranging from 50-500 nm. (C) A representative light microscopic image of HSAM nanoparticles. HSAM nanoparticles of various shape and size formed within the pores of each particle.

supernatant containing unbound doxorubicin was removed. The doxorubicin-bound HSAM nanoparticle pellet was resuspended in $20 \mu \mathrm{l}$ of $\mathrm{ddH}_{2} \mathrm{O}$ to a final concentration of $2.75 \mu \mathrm{g} / \mu \mathrm{l}$ of HSAM DNA, which contained a total of $50 \mu \mathrm{g}$ doxorubicin. The mixture was stored at $4{ }^{\circ} \mathrm{C}$ for later use.

In vitro degradation of HSAM nanoparticles. Enzymatic digestion of the doxorubicin-HSAM nanoparticles was performed in a $15-\mu 1$ reaction containing $400 \mathrm{ng}$ of HSAM DNA (with or without doxorubicin) and nuclease BAL-31 (1 U; New England Biolabs, Beverly, MA), mung bean nuclease (10 U, New England Biolabs), exonuclease I (20 U; New England Biolabs), or exonuclease III (100 U; New England Biolabs) in their associated buffers provided by the manufacturers. For DNase I, the reaction was carried out in a 15- $\mu 1$ reaction containing $40 \mathrm{U}$ DNase I (Gibco BRL, Grand Island, NY), $15 \mathrm{mM}$ Tris-Cl (8.0), $50 \mathrm{mM} \mathrm{KCl}$, and $5 \mathrm{mM}$ $\mathrm{MgCl}_{2}$. For proteinase $\mathrm{K}$, the reactions were carried out in $\mathrm{ddH}_{2} \mathrm{O}$ in the presence of $20 \mu \mathrm{g}$ proteinase $\mathrm{K}$, (Roche, Indianapolis, IN). All reactions were incubated at $37^{\circ} \mathrm{C}$ for $8 \mathrm{~h}$. At the end of incubation, an aliquot of reaction was transferred to a glass slide, and the HSAM nanoparticle was observed under a light or fluorescent microscope.

MTT assay. The percentage of growth inhibition was determined by employing an MTT (3, 4, 5-dimethylthiazol-2, 5-diphenyltetrazolium bromide; Sigma) assay to measure viable cells. A total of $5 \times 10^{3}$ cells/well were seeded onto a 96-well plate for $24 \mathrm{~h}$, treated with various amounts of doxorubicin, HSAM nanoparticles, and doxorubicin-HSAM nanoparticles, then incubated for an additional 1-5 days at $37^{\circ} \mathrm{C}$. Subsequently, $10 \mu 1$ of MTT at a concentration of $4 \mathrm{mg} / \mathrm{ml}$ was added to each well, and cells were incubated for an additional 4-6 h. The supernatant was aspirated, and $100 \mu 1$ of DMSO was added to the wells to dissolve any precipitate present. The optical density was then measured at a wavelength of $570 \mathrm{~nm}$ using an $\mathrm{EL}_{\mathrm{X}} 800$ reader (Bio-Tek Instruments, Inc.).

Animals studies. Six week-old nude mice (NCR/NU) purchased from Taconic (Cincinnati, $\mathrm{OH}$ ) were subcutaneously injected

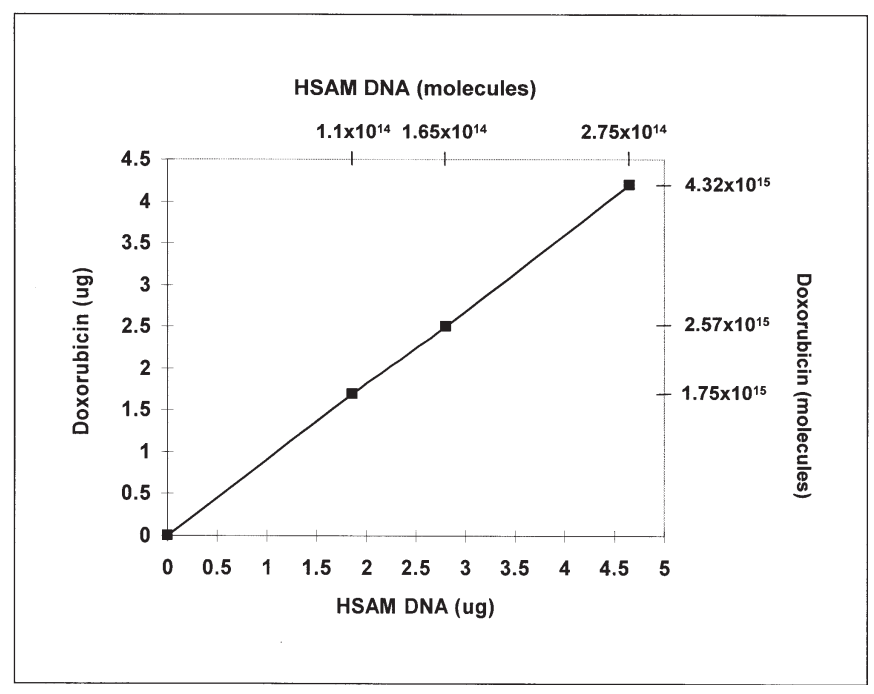

Figure 2. The binding capacity of doxorubicin. Doxorubicin $(7.5 \mu \mathrm{g})$ was added to $50 \mu 1 \mathrm{H}_{2} \mathrm{O}$ or HSAM nanoparticle containing various amounts of HSAM DNA (1.86, 2.8 and $4.7 \mu \mathrm{g})$. The absorption of supernatants at $\mathrm{OD}_{260}$ was determined using UV spectrophotometry. The amount of doxorubicin bound to HSAM nanoparticles was determined by a decrease in $\mathrm{OD}_{260}$ readings, which were taken from the HSAM nanoparticle compared to doxorubicin solution. The results showed that $1.1 \mu \mathrm{g}$ of HSAM DNA was able to carry up to $1 \mu \mathrm{g}$ of doxorubicin.

with $\mathrm{KB}$ cells $\left(5 \times 10^{6}\right)$ in the right flank. Once tumors reached approximately $100 \mathrm{~mm}^{3}$ (2 weeks), mice were randomized into two experimental groups $(n=5)$, which included a HSAM group (control) and a doxorubicin-HSAM group (treated). In the HSAM group, $20 \mu \mathrm{l}$ of HSAM nanoparticles (55 $\mu \mathrm{g}$ of HSAM DNA) were directly injected into the tumor mass twice a week for 4 weeks. In the doxorubicin-HSAM group, $20 \mu 1$ of doxorubicin-HSAM nanoparticles (55 $\mu \mathrm{g}$ of HSAM DNA) carrying $50 \mu \mathrm{g}$ of doxorubicin was injected into the tumor mass twice a week for 4 weeks. Tumor volume and weight were assessed at the beginning of each injection. Tumor volume was measured by two perpendicular dimensions (long and short) using a caliper and calculated using the formula $\left(a \times b^{2}\right) / 2$, where $a$ is the larger and $b$ is the smaller dimension of the tumor. 


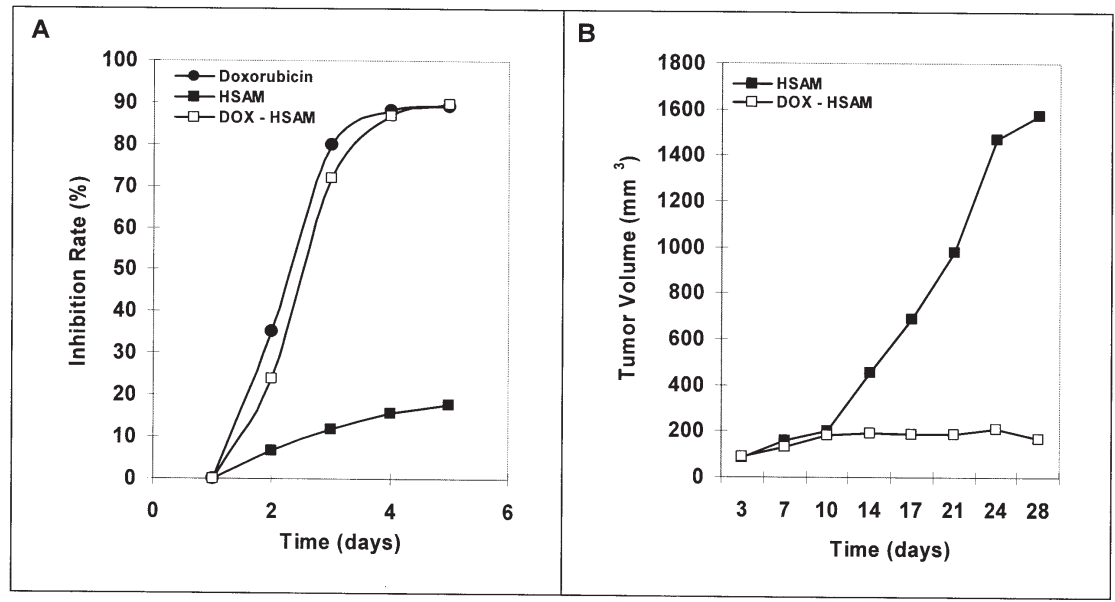

Figure 3. Inhibition of human squamous cancer cell growth. (A) In vitro inhibition of KB cell growth. KB cells were seeded onto a 96-well plate at $5 \times 10^{3}$ cells $/$ well and treated with $0.125 \mu \mathrm{g} / \mathrm{ml}$ of doxorubicin or $0.138 \mu \mathrm{g} / \mathrm{ml}$ of doxorubicin-HSAM containing $0.125 \mu \mathrm{g} / \mathrm{ml}$ of doxorubicin. The percentage of growth inhibition was determined by MTT assay on days 1-5. Each experiment was performed in triplicate. (B) Inhibition of tumor growth in nude mice bearing KB cells. Two weeks after subcutaneous inoculation of KB cells, the mice were randomly divided into two groups, 5 in each group. In the control group, $20 \mu 1$ of HSAM nanoparticles (55 $\mu \mathrm{g}$ of HSAM DNA) were injected directly into the tumor. In the treatment group, $20 \mu 1$ of doxorubicin-HSAM nanoparticle (55 $\mu \mathrm{g}$ of HSAM DNA) carrying $50 \mu \mathrm{g}$ of doxorubicin was directly injected into the tumor twice a week for 4 weeks. A significant reduction of tumor volume was observed in the treatment group after 2 weeks of treatment.

\section{Results and discussion}

Self-assembled (HSAM) nanoparticles are composed of two types of molecules, biotinylated single-stranded DNA (HSAM DNA) containing three biotins (two at each end and one central) and an avidin. The trivalent HSAM DNA are brought together by the binding of biotins to tetravalent avidins, forming a three dimensional porous structure/particle (Fig. 1A). These self-assembled particles form instantly upon mixing HSAM DNA with avidin due to the high affinity of biotin to avidin $\left(K_{\mathrm{a}}=10^{15} \mathrm{M}^{-1}\right)(6)$. Particle size can increase or decrease when the ratio of avidin to HSAM DNA is varied. For example, at a 3:4 ratio of avidin:HSAM DNA, particles as large as $10 \mu \mathrm{m}$ in diameter (microparticle) are formed. At a 1.5:4 ratio of avidin:HSAM DNA, the diameter of the HSAM particles is $<50 \mathrm{~nm}$ (nanoparticle) (Fig. 1B). At a ratio of $0.3: 4$, no particles are observed under a light or fluorescent microscope. This phenomenon is similar to the interaction between antigens (multivalent) and antibodies (bivalent) where maximal agglutination occurs when antibody concentration is high enough to approach saturation of half the binding sites of the antigen, and the resulting additional increase of antibody concentration leads to less agglutination (i.e. Heidelberger-Kendall bell-shaped relation) (7).

The ability of the HSAM nanoparticle to carry chemotherapeutic agents was tested using doxorubicin, a widely used and potent anticancer agent. Doxorubicin's mechanism of action interferes with topoisomerase II activity by intercalating into double-stranded DNA (dsDNA) between two GC base pairs (8). However, systemic toxicity, such as cardiotoxicity, mucositis and myelosuppression, limits its clinical use and administered dose $(9,10)$. We hypothesized that doxorubicin can bind to HSAM DNA (thus, HSAM nanoparticles), which can be used as a vehicle to carry doxorubicin to tumor tissue, thereby reducing its systemic toxicity. To determine whether doxorubicin can bind to the
HSAM nanoparticle, $2.5 \mu \mathrm{g}$ of doxorubicin was added to $50 \mu \mathrm{l}$ of HSAM nanoparticle (4.65 $\mu \mathrm{g}$ of HSAM DNA) and briefly incubated at room temperature. Under a light or fluorescent microscope, colorless HSAM nanoparticles became orangered because of doxorubicin's ability to absorb red light (Fig. 1C). These results indicated that doxorubicin could bind to HSAM DNA. It is interesting to note that binding of doxorubicin to the HSAM nanoparticle made the HSAM nanoparticle tighter, indicating that doxorubicin may bind to different strands of HSAM DNA and bring them closer.

To further determine the binding capacity, we measured the optical density $\left(\mathrm{OD}_{260}\right)$ value of the supernatant of the mixture of HSAM nanoparticle and doxorubicin and compared it to that of doxorubicin alone. Our results demonstrated that the amount of doxorubicin binding to the HSAM nanoparticle increased with the increase of HSAM nanoparticles (Fig. 2) and the maximal binding capacity was $1 \mu \mathrm{g}$ doxorubicin $/ 1.1 \mu \mathrm{g}$ HSAM DNA. These results indicated that approximately 15 molecules of doxorubicin can bind to each HSAM DNA or every two nucleotides can bind to one doxorubicin, which is higher than the previous prediction that every three nucleotides can bind only one doxorubicin $(8,11)$. Our results demonstrated that, although doxorubicin prefers to intercalate into dsDNA (8), it can also bind to single-stranded HSAM DNA (ssDNA). One possible mechanism is that doxorubicin brings together two strands of HSAM DNA, which is consistent with the observation that doxorubicin causes the HSAM nanoparticle to become more compact. We further tested the binding of stable doxorubicin to HSAM DNA. Doxorubicin-HSAM nanoparticles were incubated in $\mathrm{ddH}_{2} \mathrm{O}$ for 3 days at room temperature, and the $\mathrm{OD}_{260}$ value of the supernatant was determined. Our results showed no significant increase in $\mathrm{OD}_{260}$ value, indicating that doxorubicin irreversibly binds to HSAM DNA.

In order for HSAM nanoparticles to carry doxorubicin as a therapeutic agent, doxorubicin has to be released from the 
HSAM DNA. Possible biological releasing agents could be proteases that break down avidin and nucleases that break down HSAM DNA. The doxorubicin-HSAM nanoparticles were incubated with proteinase $\mathrm{K}$ (a broad-spectrum endopeptidase), DNase I (an endonuclease that digests ssDNA and dsDNA), exonuclease I (digests ssDNA from the $3^{\prime}$ end), exonuclease III (digests dsDNA from the 3 ' end), mung bean nuclease (an exonuclease that digests the ssDNA region of dsDNA from both the $3^{\prime}$ and $5^{\prime}$ end) and nuclease BAL-31 (single-stranded specific endodeoxyribonuclease with $3^{\prime}$ and $5^{\prime}$ exonuclease activity). Under a light or fluorescent microscope, both the HSAM and doxorubicinHSAM nanoparticles decreased after incubation with nuclease BAL-31 and DNase I with similar efficiencies. These results indicated that nucleases gained access to HSAM DNA, and doxorubicin did not interfere with the digestion of HSAM DNA. However, no distinct change in size and/or number of doxorubicin-HSAM nanoparticles was noticed after incubation with other exonucleases. These results indicated that both ends of the HSAM DNA were unavailable for exonuclease activity, probably due to the presence of biotin moieties. In addition, no obvious changes were observed after incubation with proteinase $\mathrm{K}$, which is perhaps due to the inaccessibility of proteinase $\mathrm{K}$ to HSAM nanoparticles or protection of avidin from degradation by HSAM DNA.

Although HSAM nanoparticles can be degraded by nucleases, doxorubicin released from the HSAM particle may not be biologically active. Therefore, we tested the ability of doxorubicin-HSAM nanoparticles to inhibit cancer cell growth in vitro. The hypothesis was that cancer cells could engulf HSAM nanoparticles and intracellularly degrade them via nucleases. Alternatively, necrotic cancer cells release a large amount of nucleases into pericellular space, which may also degrade HSAM nanoparticles. The in vitro viability of $\mathrm{KB}$ cells (a human oral squamous cancer cell line) was determined by MTT assay in the presence of doxorubicin, doxorubicin-HSAM nanoparticles and HSAM nanoparticles. Our results showed that doxorubicin alone or doxorubicinHSAM nanoparticles were able to inhibit KB cell growth and reached $90 \%$ inhibition on day 5 (Fig. 3A). These results indicate that doxorubicin released from HSAM nanoparticles maintained its anticancer activity. However, a 3-h delay of growth inhibition was observed with doxorubicin-HSAM nanoparticles compared with doxorubicin alone (at an equal concentration of doxorubicin). The delay in cancer cell growth inhibition by doxorubicin-HSAM nanoparticles was probably due to the delayed release of doxorubicin from the HSAM nanoparticles.

To confirm the anticancer activity of doxorubicin-HSAM particles, we compared tumor growth inhibition in nude mice bearing KB cells by direct intratumoral injections of HSAM nanoparticles and doxorubicin-HSAM nanoparticles. Our results showed that doxorubicin-HSAM nanoparticles suppressed tumor growth for up to 8 weeks. The dose required to suppress tumor growth by direct injection of doxorubicinHSAM nanoparticles into the tumor was much less than that required by systemic administration (i.p. or i.v.) (12). Furthermore, the concentration of doxorubicin in tumor tissue reached much higher levels than that with systemic delivery of free doxorubicin. A previous study demonstrated that a single dose of doxorubicin injected through the mouse tail vein can only reach $1 \mathrm{ng} / \mathrm{mg}$ in tumor tissue (13), while doxorubicin delivered to the tumor by the HSAM nanoparticle was $180 \mathrm{ng} / \mathrm{mg}$, 180fold higher. These results indicated that HSAM can serve as a matrix to maintain the drug locally and prevent doxorubicin from being carried away by blood flow, thus achieving a higher intratumoral concentration and reducing systemic toxicity. Upon histological examination, no viable cancer cells were observed in any of the tumors treated with doxorubicinHSAM particles and the tumors were completely replaced with fibrosis, indicating complete suppression of cancer cell growth. Furthermore, HSAM nanoparticles were not identified in tumor tissue, which is indicative of complete degradation. These results signify that doxorubicin-HSAM nanoparticles can be degraded either intracellularly after endocytosis by tumor cells or in the pericellular space. No toxicity was observed in any animal since the dose $(2.5 \mathrm{mg} / \mathrm{kg})$ injected into the tumor mass is much less than the dose required to produce systemic toxicity $\left(\mathrm{LD}_{50}\right.$ for free doxorubicin via i.p. was $12 \mathrm{mg} / \mathrm{kg}$ ) (14).

HSAM nanoparticles represent a novel drug delivery system. HSAM nanoparticles can be easily assembled by mixing biotinylated DNA and avidin. The size and shape of HSAM nanoparticles can easily be controlled by adjusting the number of biotins in the HSAM DNA and the ratio of HSAM DNA to avidin. The porous structure of the HSAM nanoparticle is highly permeable, allowing both small and large molecules to be easily packed into the particles. The binding of doxorubicin to HSAM nanoparticles is consistent and stable and does not require a complex chemistry as in other polymer-based drug delivery systems (15). In addition to doxorubicin, other intercalating anticancer drugs, such as daunorubicin, aclacinomycin and mitomcyin (10), can be delivered by the HSAM nanoparticle. In this study, we demonstrated that direct injection of the doxorubicin-HSAM nanoparticle into the tumor mass can inhibit tumor growth. This approach has a significant clinical implication. Direct percutaneous injection of anticancer agents into the tumor has been used to treat several solid tumors, such as hepatocellular carcinoma, under ultrasound guidance $(16,17)$ or superficial head and neck cancers by needle injection (18). Slower and localized release of anticancer agents can make this approach more effective than current systemic modalities. The rate of drug release can easily be controlled by the level of resistance of the HSAM nanoparticle to nucleases. For example, a phosphorothioate moiety can be introduced into the HSAM DNA that will increase its resistance to nuclease (19). Another advantage of the HSAM nanoparticle is that it can be used for systemic delivery of doxorubicin because of its small size and temporally controlled release. Targeted delivery of doxorubicin-HSAM nanoparticles to tumor tissue after systemic administration can be achieved by attaching a tumor-specific antibody to the HSAM nanoparticle through biotins attached to the antibody and the avidin of the HSAM nanoparticles (15).

In summary, we demonstrated that a novel biocompatible and biodegradable self-assembled nanoparticle can be used as a vehicle to carry a highly toxic anticancer drug to its target tissues. Therefore, a higher local drug concentration and reduction in systemic toxicity is attained. 


\section{Acknowledgements}

This work was supported in part by a grant from Hamilton Thorne Biosciences to D.Y.Z. The authors would like to acknowledge the critical review and comments by Drs Harry McCoy, David Lane and Amy Wilson.

\section{References}

1. Heidenreich A, Sommer F, Ohlmann C, Schrader A, Olbert P, Goecke J and Engelmann U: Prospective randomized phase II trial of pegylated doxorubicin in the management of symptomatic hormone-refractory prostate carcinoma. Cancer 101: 948-956, 2004.

2. Campbell R, Balasubramanian S and Straubinger R: Influence of cationic lipids on the stability and membrane properties of paclitaxel-containing liposomes. J Pharm Sci 90: 1091-1105, 2001.

3. Sharma A, Mayhew E, Bolcsak L, Cavanaugh C, Harmon P, Janoff A and Bernacki R: Activity of paclitaxel liposome formulations against human ovarian tumor xenografts. Int J Cancer 71: 103-107, 1997.

4. Shuai X, Ai H, Nasongkla N, Kim S and Gao J: Micellar carriers based on block copolymers of poly( $\varepsilon$-caprolactone) and poly(ethylene glycol) for doxorubicin delivery. J Control Release 98: 415-426, 2004.

5. Aulenta F, Hayes W and Rannard S: Dendrimers: a new class of nanoscopic containers and delivery devices. Eur Polym J 39: 1741-1771, 2003.

6. Livnah O, Bayer EA, Wilchek M and Sussman JL: Threedimensional structures of avidin and the avidin-biotin complex. Proc Natl Acad Sci USA 90: 5076-5080, 1993.

7. Heidelberger M and Kendall FW: Quantitative theory of the precipitation reaction; study of azoprotein-antibody system. J Exp Med 62: 467-483, 1935.

8. Yang XL and Wang AH: Structural studies of atom-specific anticancer drugs acting on DNA. Pharmacol Ther 83: 181-215. 1999.
9. Forrest GL, Gonzalez B, Tseng W, Li X and Mann J: Human carbonyl reductase overexpression in the heart advances the development of doxorubicin-induced cardiotoxicity in transgenic mice. Cancer Res 60: 5158-5164, 2000.

10. Richardson DS and Johnson SA: Anthracyclines in haematology: preclinical studies, toxicity and delivery systems. Blood Rev 11: 201-223, 1997.

11. Wang AHJ: Intercalative drug binding to DNA. Curr Opin Struct Biol 2: 361-368, 1992.

12. Moase EH, Qi W, Ishida T, et al: Anti-MUC-1 immunoliposomal doxorubicin in the treatment of murine models of metastatic breast cancer. Biochim Biophys Acta 1510: 43-55, 2001

13. Kong G, Anyarambhatla G, Petros WP, Braun, RD, Colvin OM, Needham D and Dewhirst MW: Efficacy of liposomes and hyperthermia in a human tumor xenograft model: importance of triggered drug release. Cancer Res 60: 6950-6957, 2000.

14. Trouet A, Passioukov A, Van der Poorten K, et al: Extracellularly tumor-activated prodrugs for the selective chemotherapy of cancer: application to doxorubicin and preliminary in vitro and in vivo studies. Cancer Res 61: 2843-2846, 2001.

15. Kovar M, Strohalm J, Etrych T, Ulbrich K and Rihova B: Star structure of antibody-targeted HPMA copolymer-bound doxorubicin: a novel type of polymeric conjugate for targeted drug delivery with potent antitumor effect. Bioconjug Chem 13: 206-215, 2002.

16. Leung TW, Yu S, Johnson PJ, et al: Phase II study of the efficacy and safety of cisplatin-epinephrine injectable gel administered to patients with unresectable hepatocellular carcinoma. J Clin Oncol 21: 652-658, 2003.

17. Nakamura $\mathrm{S}$ and Tanaka K: Combination of transcatheter arterial embolization and percutaneous ethanol injection for hepatocellular carcinoma: comparison of efficacies of doxorubicin and epirubicin in transcatheter arterial embolization. Semin Oncol 24: S6-S49, 1997.

18. Lamont JP, Nemunaitis J, Kuhn JA, Landers SA and McCarty TM: A prospective phase II trial of ONYX-015 adenovirus and chemotherapy in recurrent squamous cell carcinoma of the head and neck (the Baylor experience). Ann Surg Oncol 7: 588-592, 2000.

19. Kausch I and Bohle A: Antisense oligonucleotide therapy in urology. J Urol 168: 239-247, 2002. 